\title{
Visual Direction Indication for Ships
}

\author{
J. I. Potts \\ (Assistant Inspector of Shipping, Nigeria)
}

THERE is no doubt that everyone is agreed that a combination of visual and sound signals are a necessity, but, in view of the large disparity in the speeds of light and sound, the method of achieving this has not really been agreed upon, although a few countries supported the system of synchronizing the light and sound signals, thus bringing about the addition of Rule $28(\mathrm{c})$ to the Collision Regulations. I am, however, of the opinion that this synchronized system is not suitable, mainly because of the disparity between the relative speeds of light and sound. This could lead to confusion and misinterpretation by the receiving vessel; the degree of confusion depending on how far distant the receiving vessel is from the vessel giving the signals, when there is a sudden alteration of course immediately following the previous alteration:

In Circular No. 3717 of the Royal Netherlands Shipowners' Association, 'Recommendation on Trafficators at Sea' (Journal 19, 267), comparison was made with car traffic on the road, and it is pointed out that indication of a change in direction, given by means of an optical signal (flasher-lights these days), is already compulsory for all motor vehicles. I see no reason why the same system of flasher-lights cannot be applied to ships with, of course, a few differences, to be used in conjunction with sound signals, but not synchronous with them. In fact, it is better that the light and sound signals are not synchronous, with the system that I propose.

In conjunction with the sound signals required to be given in accordance with Rule 28(a) of the Collision Regulations, a flashing light should also be shown indicating the relevant action being taken, viz. a green flashing light in conjunction with one short blast, a red flashing light in conjunction with two short blasts, and a white flashing light in conjunction with three short blasts. Whereas the sound signals are only required to be given once, at the beginning of the manœuvre, these flashing lights may be left in operation until the manœuvre has been completed and the vessel steadied on the new course, thus indicating the full extent of the mancuvre. These lights may be placed in a vertical line, one over the other, situated on top of, and operated from, the wheelhouse; and they should be visible all round the horizon at a distance of at least 5 miles.

In view of the foregoing, therefore, I should like to suggest that Rule 2 (a) be amended by the addition of the following:

(vii) On top of the wheelhouse, where they can best be seen, three lights in a vertical line one over the other not less than $6 \mathrm{ft}$. apart., and of such a character as to be visible all round the horizon at a distance of at least 5 miles. The highest of these lights shall be white in colour, the middle one shall be green, and the lowest one shall be red, and each of these lights shall have continuous flashing characteristics, and each shall be operated independently of the others in accordance with Rule 28 (c).

and Rule 28 (c) be amended as follows:

'Any whistle signal mentioned in this Rule shall be further indicated by a 
visual signal consisting of one of the lights mentioned in Rule 2(a) (vii) in the following manner:

I. The green light when one short blast is given.

2. The red light when two short blasts are given.

3. The white light when three short blasts are given.

The appropriate light shall be left in operation until the relevant manœuvre has been completed.' 